\title{
Review and prospect of compressed air energy storage system
}

\author{
Laijun CHEN ${ }^{1}$, Tianwen ZHENG $^{1}$, Shengwei MEI ${ }^{1}$, Xiaodai XUE ${ }^{1}$, \\ Binhui LIU ${ }^{1}$, Qiang LU ${ }^{1}$
}

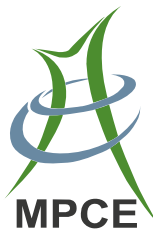

\begin{abstract}
As an effective approach of implementing power load shifting, fostering the accommodation of renewable energy, such as the wind and solar generation, energy storage technique is playing an important role in the smart grid and energy internet. Compressed air energy storage (CAES) is a promising energy storage technology due to its cleanness, high efficiency, low cost, and long service life. This paper surveys state-of-the-art technologies of CAES, and makes endeavors to demonstrate the fundamental principles, classifications and operation modes of CAES. Critical subsystems of CAES are elaborated exhaustively. The application prospects and further research directions are summarized to promote the popularization of CAES in smart grid and energy internet.
\end{abstract}

CrossCheck date: 13 September 2016

Received: 19 July 2016/Accepted: 14 September 2016/Published online: 15 October 2016

(C) The Author(s) 2016. This article is published with open access at Springerlink.com

$\triangle$ Xiaodai XUE

xuexiaodai@tsinghua.edu.cn

Laijun CHEN

chenlaijun@tsinghua.edu.cn

Tianwen ZHENG

tianwenscu@163.com

Shengwei MEI

meishengwei@tsinghua.edu.cn

Binhui LIU

liubhsophia@outlook.com

Qiang LU

luqiang@tsinghua.edu.cn

1 Department of Electrical Engineering, Tsinghua University, Beijing 100084, China
Keywords Energy storage, Compressed air energy storage (CAES), Smart grid, Energy internet

\section{Introduction}

The development and utilization of renewable energy is an important remedy for the worldwide fossil energy crisis and environmental pollution issues [1]. Due to the volatility and randomness of renewable energies, such as the wind and solar power, integration of such energy resources into power grid imposes great challenges on the secure operation and power quality of modern power systems [2]. Thus, how to utilize renewable energies in a safe, efficient and economical way has gained much attention.

Energy storage technology is an important means to solve the above problems. With the capability of reshaping the load profile, energy storage system (ESS) adds additional flexibility on system operation and helps utilize large-scale renewable energy [3]. Meanwhile, large-scale energy storage technology can reduce the gap between peak and valley loads to enhance the efficiency of generation assets. Undoubtedly, ESS plays an important role in smart grid and energy internet and becomes a hot topic in the field of energy research $[4,5]$.

There are several mature energy storage technologies, including chemical battery energy storage, pumped storage and compressed air energy storage (CAES) [4, 5]. Among them, chemical battery energy storage technology is the most popular one, but the investment and recycling cost, as well as potential environmental problems limit its largescale application. Pumped storage is widely applied in the area of abundant water resource. However, special geographical conditions restrict the available site and potential ecological damage prevents further popularization of 
pumped storage. With the features of fewer construction constraints, high efficiency and environmental friendliness, CAES is expected to be one of the most promising energy storage technologies to address many problems encountered by the high penetration of renewable energy in smart grid and energy internet.

The effectiveness of hybrid energy storage by combing micro CAES with flywheel ESS is modeled and verified in [6]. CAES and super capacitors based hybrid ESS is also verified in [7]. The joint operation of power system considering pumped storage and distributed CAES is investigated in [8].

There exists two large-scale CAES power plants, including Huntorf and McIntosh, which are under commercial operation, and many other CAES plants are under construction $[5,9]$. In addition, a number of research institutions and companies have proposed many innovative CAES architectures. Institute of Engineering Thermophysics, Chinese Academy of Sciences (IET-CAS), began to study supercritical CAES technology by combining regular CAES technology and liquid air energy storage (LAES) technology from 2009. By far, IET-CAS has completed a $15 \mathrm{~kW}$ experimental system and a 1.5 MW demonstration system $[10,11]$. Under the fund of the State Grid key technology project, Tsinghua University, China Electric Power Research Institute (CEPRI) and Technical Institute of Physics and Chemistry of the Chinese Academy of Sciences have constructed a $500 \mathrm{~kW}$ non-supplementary fired CAES (NSF-CAES) and finished the field tests in 2014 [12]. Owing to the promising prospects of CAES technology, Bill Gates invested the CAES project of LightSail Energy, a famous CAES company, which attracts many people to pay much more attention to CAES technology.

Undoubtedly, CAES technology is still in the early stage of development. To understand CAES technology deeply and explore future application directions, this paper introduces the fundamental principles and the state of the art of CAES technology, and analyzes the related key technologies and research progress. Application prospects of CAES in smart grid and energy internet are also depicted to promote the development and application of CAES technology.

\section{CAES techniques}

\subsection{Fundamental principle}

CAES is an energy storage technology based on gas turbine technology, which uses electricity to compress air and stores the high-pressure air in storage reservoir by means of underground salt cavern, underground mine, expired wells, or gas chamber during energy storage period, and releases the compressed air to drive turbine to generate electricity during the stage of power supply [13, 14]. In simple terms, the charge life of CAES depends on its mechanical level, which means it is not easy to become fatigue as the battery. Normally, the life of CAES is 30 to 40 years.

During charging process, the compressor utilizes offpeak electricity, curtailed wind and solar power to compress ambient air. The consumed power of each compression stage is given by:

$P_{\mathrm{c}, i}=\frac{k}{k-1} \frac{Q_{\mathrm{m}, \mathrm{c}} R_{\mathrm{g}} T_{\mathrm{c}, i}^{\mathrm{in}}}{\eta_{\mathrm{c}, i}}\left[\left(\beta_{\mathrm{c}, i}\right)^{\frac{k-1}{k}}-1\right]$

where $k$ is adiabatic exponent; $Q_{\mathrm{m}, \mathrm{c}}$ is the mass flow rate of air; $R_{\mathrm{g}}$ is air gas constant; $T_{\mathrm{c}, i}^{\mathrm{in}}$ is the air temperature inlet of compressor; $\eta_{\mathrm{c}, i}$ is efficiency of compressor; and $\beta_{\mathrm{c}, i}$ is compression ratio.

The air temperature of outlet of each compression stage can be expressed as:

$T_{\mathrm{c}, i}^{\text {out }}=T_{\mathrm{c}, i}^{\text {in }}\left[\frac{\left(\beta_{\mathrm{c}, i}\right)^{\frac{k-1}{k}}-1}{\eta_{\mathrm{c}, i}}+1\right]$

where $T_{\mathrm{c}, i}^{\text {out }}$ is the air temperature outlet of compressor.

For an $n$-stage compressor and a $t_{\mathrm{c}}$ charging time, the total consumed electricity of compressor can be calculated as:

$W_{\mathrm{c}}=\eta_{\mathrm{a}} \sum_{i=1}^{n} P_{\mathrm{c}, i} t_{\mathrm{c}}$

where $\eta_{\mathrm{a}}$ is the efficiency of air-compressor.

Utilizing adiabatic efficiency to compute the actual shaft power of turbine, power of each turbine satisfies:

$P_{\mathrm{e}, i}=\frac{k}{k-1} Q_{\mathrm{m}, \mathrm{e}} R_{\mathrm{g}} T_{\mathrm{e}, i} \eta_{\mathrm{e}, i}\left[1-\left(\pi_{\mathrm{e}, i}\right)^{-\frac{k-1}{k}}\right]$

where $Q_{\mathrm{m}, \mathrm{e}}$ is mass flow rate of turbine; $\eta_{\mathrm{e}, i}$ is turbine efficiency; and $\pi_{\mathrm{e}, i}$ is expansion ratio of turbine.

For an $m$-stage turbine, with a $t_{\mathrm{e}}$ discharging time, the total output electricity can be calculated by:

$W_{\mathrm{e}}=\eta_{\mathrm{g}} \sum_{i=1}^{m} P_{\mathrm{e}, i} t_{\mathrm{e}}$

where $\eta_{\mathrm{g}}$ is the efficiency of generator.

\subsection{Classification}

Up to now, various CAES architectures have been developed. According to the criterion of whether fuel combustion is needed or not, the CAES can be classified into supplementary fired CAES (SF-CAES) and NSF-CAES.

\section{1) SF-CAES}

Except for the fundamental structures of CAES, the SFCAES architecture needs fuel combustion during energy 


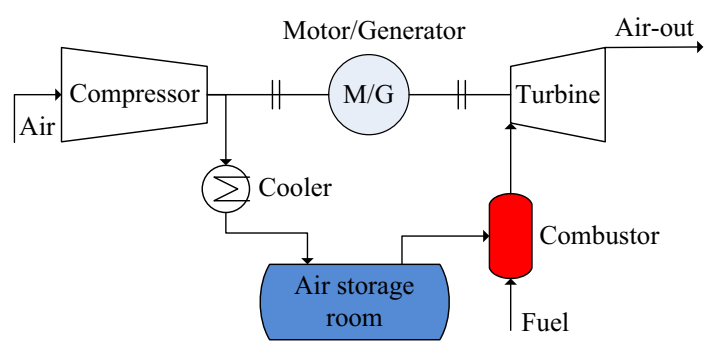

Fig. 1 Diagram of SF-CAES system

discharging process to achieve cycle operation as in Fig. 1.

SF-CAES technique is established on the principle of division of compression and expansion process of gas turbine generator. Compressor and turbine of gas turbine are usually operated simultaneously, whereas those of SFCAES work separately. Meanwhile, the compressor of gas turbine consumes a certain part of the power generated by turbine to compress ambient air. However, there is no need to consume power generated by turbine during air compression phase of SF-CAES. In this regard, the SF-CAES system has a strong dependence on fossil fuel as well as environment pollution due to the combustion of gas.

\section{2) NSF-CAES}

Distinguished from SF-CAES, NSF-CAES collects, stores and re-uses compression thermal to heat compressed air entering the turbine based on heat regeneration technology. Typical design schemes of NSF-CAES system include non-adiabatic compression without supplementary fired, adiabatic compression with one-stage thermal energy recycling and adiabatic compression with multi-stage thermal energy recycling. Diagram of NSF-CAES system is depicted in Fig. 2.

NSF-CAES is independent of fuel by replacing the supplementary fuel firing with compression heat, thus, no harmful gas is generated. This kind of system can be used for heating with stored compression heat and for cooling with low-temperature air exhausted from the turbine. NSFCAES can supply cold, heat and electricity simultaneously, which implements energy comprehensive utilization and improves system efficiency.

NSF-CAES mentioned above usually adopts gaseous air as the recycling medium, LAES is another special NSFCAES technique with liquid air as the recycling medium. LAES uses stored cold energy to cool and liquefy the compressed high-pressure air, and stores it in a low-temperature adiabatic container during the charging process. During the on-peak time, the liquid air absorbs thermal using the heat exchanger after pressurizing, and the released cold in the gasification process can be recycled and stored, then high-pressure air absorbs the stored

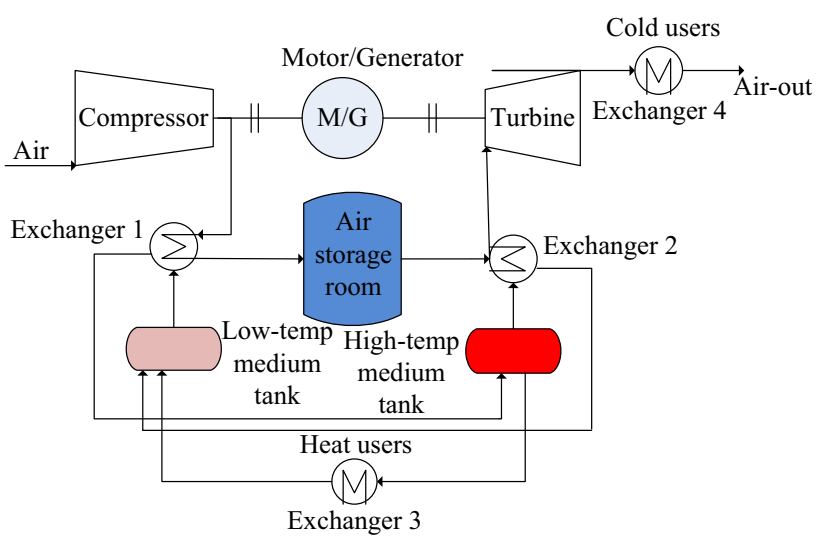

Fig. 2 Diagram of NSF-CAES system

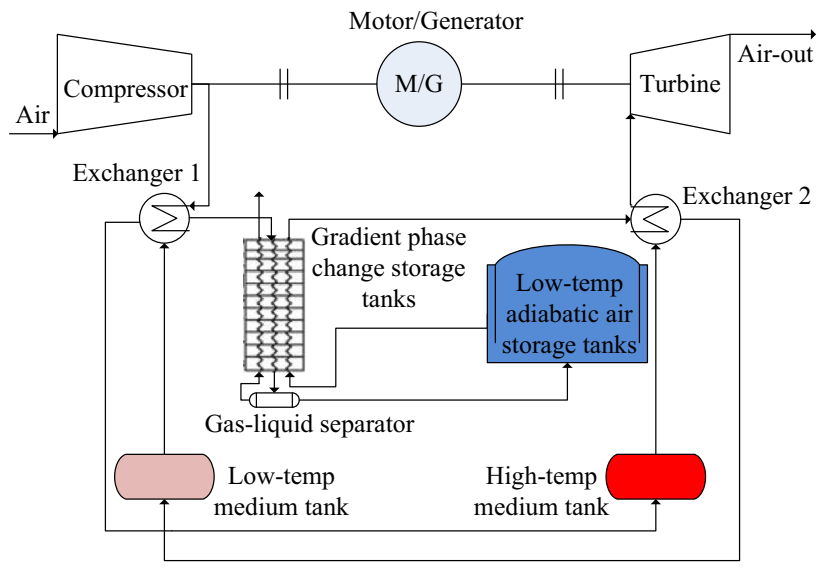

Fig. 3 Diagram of LAES system

compression heat before being sent into turbine, and then drives the motor to generate electricity. Diagram of the LAES system is illustrated in Fig. 3.

\subsection{CAES development status}

\section{1) Large scale CAES system}

A CAES system with more than 50 MW scale capacity could be categorized as a large scale one [14]. There exists two commercial CAES plants, including Huntorf plant located in Germany and McIntosh plant in the United States. Huntorf CAES plant was finished in 1978, and is still operating. The compressor power and output power of Huntorf are 60 and $321 \mathrm{MW}$, respectively. The Huntorf unit can be charged continuously for $8 \mathrm{~h}$ to provide a $2 \mathrm{~h}$ power generation $[15,16]$. Huntorf plant adopts the SFCAES architecture, the compressed air is stored in a $600 \mathrm{~m}$ depth mined salt cavern with a $310000 \mathrm{~m}^{3}$ volume, and the storage pressure is in the range from 5 to $7 \mathrm{MPa}$ [15]. The electricity to electricity efficiency can reach $42 \%$ with natural gas combustion, and the total electricity generated 
yearly reached $294465 \mathrm{MWh}$ in 2007 [17]. With the SFCAES architecture, Huntorf plant has a strong dependence on natural gas, the power generation along with lots of carbon emission. The total carbon emission in 2007 reached $156540 \mathrm{t}$ [17], which opposites the low-carbonemission requirement of modern low carbon society.

McIntosh CAES plant, which was built in 1991 and located in Alabama, is the second commercial CAES plant. It consists of a $50 \mathrm{MW}$ compressor and a $110 \mathrm{MW}$ power generation unit, and is designed for a continuous $41 \mathrm{~h}$ air compression and $26 \mathrm{~h}$ power supplying [16]. The underground gas storage cavern is located in a $450 \mathrm{~m}$ rock salt layer with the operation pressure from 4.5 to $7.4 \mathrm{MPa}$ [16]. Unlike Huntorf plant, McIntosh CAES plant uses exhaust high-temperature air of the last stage of gas turbine to preheat the inlet of gas turbine through a heat exchanger. Therefore, the system thermal efficiency is increased by $25 \%$, while the whole system efficiency is improved to $54 \%$. However, similar to Huntorf plant, McIntosh plant still uses SF-CAES architecture, which increases the risk of environmental pollution.

It should be explained that the research process of large scale CAES is not smoothly developed. A number of CAES systems have been aborted due to various reasons. The 270 MW Iowa CAES, began in 2003, was suspended in 2011 due to geological conditions and economic reasons [18]. The $270 \mathrm{MW}$ Ohio CAES, began in 2009, was announced being suspended in 2013 due to economic considerations [19]. Therefore, system construction cost is one of the main factors limiting CAES development.

With the increasing demand of ESS in smart grid, several advanced CAES architectures have been developed. RWE Power, which is the biggest German power company, launched the ADELE CAES plant based on the adiabatic compression with one-stage thermal recycling technique in 2010 [20]. The outlet air from compressor during energy storage process is expected to reach $600{ }^{\circ} \mathrm{C}$ and $10 \mathrm{MPa}$ [21]. Heat storage device is implemented to store the air compression thermal. The collected thermal is utilized to heat the inlet air of turbine during energy discharging process, and to improve the power generation capability. Noting that, the designed ADELE plant has a $90 \mathrm{MW}$ turbine, and the designed whole efficiency is expected to reach $70 \%$. However, it is no longer expected to be built due to economic issues.

\section{2) Small scale CAES system}

Except for the $50 \mathrm{MW}$ scale large CAES system, several companies and institutes have investigated small scale CAES systems with less than $50 \mathrm{MW}$ capacity [14].

The 2 MW Kamisunagawa CAES located in Sorachi District, Hokkaido, began to construct in 1998 and has been put into operation since 2001. This CAES system utilizes an underground rock structure hole with about $400 \mathrm{~m}$ depth and about $1600 \mathrm{~m}^{3}$ volume to store the compressed air, and the maximum pressure reaches $8 \mathrm{MPa}$. The Kamisunagawa system is a SF-CAES.

The leader of LAES technique, Highview Power Storage located in British, has established a LAES pilot plant with a $350 \mathrm{~kW} / 2.5 \mathrm{MWh}$ capacity at Slough, Greater London. The plant has been operated and connected to UK state grid since July 2011 [22]. The conversion efficiency from electricity to electricity is about $8 \%$ [22]. It is worth mentioning that the low-temperature energy system of Highview utilizes the surplus energy to transform the air to a liquid one and stores the air into an adiabatic container with low-temperature. During on-peak time, the liquid air is discharged to a closed space with a high-temperature to a vaporization one, and the turbine is driven to generate electricity. The system efficiency is expected to be improved from $25 \%$ to $70 \%$ by combining the LAES system with a device generating waste thermal, which can be used to liquefy air. A pre-commercial 5 MW LAES pilot plant is under construction in Pilsworth, Greater Manchester, and a conceptual $200 \mathrm{MW} / 1.2 \mathrm{GWh}$ LAES plant is also proposed by Highview [22]. Moreover, the second stage of the Wind-Solar-Storage-Transmission project in Zhangbei, China, intended to use the LAES technique toward a low carbon Winter Olympics.

Lightsail Energy, established in 2009, dedicated to small size, modularized CAES solutions and the production of storage tanks for pressurized gases. The product mainly utilized an invertible reciprocating composite piston compressor/expander, with a capacity of $250 \mathrm{~kW}$ [23]. Lightsail Energy employed its first ESS at the end of 2015, and plans to deploy another four systems during 2016. The heat efficiency of Lightsail Energy product is expected to reach $90 \%$. Its first generation product, Lightsail Energy V.1, enables renewable energy resources to provide cheaper electricity than that produced by diesel generators [23]. The second generation product, Lightsail Energy V.2, is expected to be the first ESS to outcompete gas turbine peaking-load power plants [23].

Sustain X, an America CAES company, has obtained breakthrough developments in the field of isothermal CAES (ICAES). The company started to build the world's first MW-scale ICAES system from 2013, the system has a capacity of $1.5 \mathrm{MW}$ and is still under construction [24].

Recently, China has begun to focus on CAES technology. IET-CAS has focused on supercritical CAES technology since 2009. This institute integrated the traditional CAES and liquid air storage to build a $1.5 \mathrm{MW}$ supercritical CAES demonstration system. It has been operated more than $168 \mathrm{~h}$ during experiments [25]. However, the system has not finished the conversion process from electricity to electricity, i.e., it lacks the power generation 
process. At present, this institute is launching the research of a $10 \mathrm{MW}$ advanced CAES system.

In 2014, Tsinghua University developed a 500 kW NSFCAES demonstration system, i.e., TICC-500. TICC-500 utilizes a NSF-CAES framework, and has completed the experiment of joint supplying of cool, thermal and electricity. It has the advantages of zero-carbon-emission, high efficiency and flexible operation. The air pressure is in the range from 3 to $11 \mathrm{MPa}$. TICC-500 uses a $5.5 \mathrm{~h}$ charging process during the off-peak time to realize a $1 \mathrm{~h}$ discharging process during the on-peak time. The volume of storage tank is $100 \mathrm{~m}^{3}$, and electric-to-electric efficiency is up to $33 \%[12,26]$. There are various factors influencing the efficiency of TICC-500, the efficiency can be enhanced through optimizing key parameters of the system as analyzed in [27]. Furthermore, TICC-500 adopts water as thermal energy storage (TES) medium to recycle the heat. The efficiency can be further improved and results in a competitive market if heat conducting oil and molten salt could be used as TES medium. Meanwhile, the Tsinghua CAES team established a $100 \mathrm{~kW}$ concentrating solar hybrid NSF-CAES system, which is called CSHC-100, by using trough collectors to collect solar thermal in Qinghai University. Besides, the team has designed a 50 MW NSFCAES with a salt carven air storage system and pipeline steel based $10 \mathrm{MW}$ NSF-CAES for Jintan, Jiangsu and Haixi, Qinghai, which is based on the geographic conditions of these two areas. The electricity to electricity conversion efficiency is expected to reach $52.4 \%$ and $50.3 \%$, respectively, which are adequate for commercial operation.

Although China and other countries have explored both large-scale and small-scale CAES systems, key technical breakthroughs related to the compression, air storage, thermal storage and turbine power generation are still desired, and the efficiency of most current CAES plants can be improved. To further enhance the efficiency market competitiveness of CAES, new thermal technology and progress in material science are of great importance.

\section{Key subsystems of CAES}

In terms of function and principle, CAES is mainly composed of several key subsystems, including compression subsystem, air storage subsystem, heat regeneration subsystem, and electric power generation subsystem. The compression subsystem uses surplus electricity to drive compressors to produce high-pressure air, along with the high-temperature compression thermal energy. The air storage subsystem is used to store high-pressure air generated by the compressor, thus, to store the molecular potential energy simultaneously. During the expansion process, the heat regeneration subsystem heats the highpressure air to improve the entrance enthalpy of starting point of air expansion. The electric power generation subsystem is implemented to drive the turbine to generate electricity with high-temperature air, therefore, finishing the conversion from molecular potential energy and thermal energy to electricity. It is worth mentioning that the compressor is the electrical interface for storing energy, and the generator is the electrical interface for supplying energy. More details of each subsystem are elaborated as follows.

\subsection{Compression subsystem}

Compression subsystem is the core part of CAES. The compressor has the characteristics of large air mass flow rate, high efficiency, high compression ratio, and large backpressure change, which is a big difference from the compressor in gas turbine and general industrial compressor [28-30]. There are a variety of air compression schemes according to size and operating principles of the CAES system. Deployments of compression subsystem of typical CAES plants are illustrated in Table 1.

The structure of multi-stage compression, inter-stage and post-stage cooling composed of axial compressor in low-pressure stage, and centrifugal compressor in highpressure stage has been adopted frequently in the design of large scale CAES power plant. This compression scheme is mature in terms of designing and manufacturing and has been adopted by Huntorf and McIntosh CAES plants [5]. However, for the advanced adiabatic CAES (AA-CAES), the axial or centrifugal compressor, which processes better performance, large pressure ratio and high temperature rise should be deployed to ensure the high adiabatic compression efficiency to suffer from higher pressure ratio and exhaust temperature, as utilized in the ADELE NSF-CAES started in 2010 and progresses slowly because of the immature technology and investment cost issue [2].

For the small scale CAES, as the high requirement for space flexibility, the pressure of air storage system is required to be high enough to reduce the volume of the air

Table 1 Compression subsystem of typical CAES plants

\begin{tabular}{ll}
\hline Plant & Structure of compressor \\
\hline Huntorf & $\begin{array}{r}\text { Low pressure section axial, high pressure section } \\
\text { centrifugal compressor }\end{array}$ \\
McIntosh & $\begin{array}{r}\text { Low pressure section axial, high pressure section } \\
\text { centrifugal compressor }\end{array}$ \\
ADELE & $\begin{array}{c}\text { Axial flow compressor with large compression ratio, } \\
\text { high temperature increase }\end{array}$ \\
TICC-500 & 5-stage reciprocating compressor \\
Sustain X & 2-stage reciprocating compressor \\
\hline
\end{tabular}


storage tank. Meantime, the air mass flow rate of the system is relatively small. Thus it is more appropriate to use single-stage or multi-stage reciprocating compressor, however, the invest cost is also high.

Several factors need to be taken into account during the design of compression flowchart. Some of them are listed as follows.

1) The compressor works at an unsteady state during the whole operation.

2) The compression process is required to approximate adiabatic compression to reduce thermal energy loss.

3) Outlet air temperature of each compressor is assumed to be close to dispatch the thermal ESS.

4) Potential safety issue of air cylinder due to the higher outlet temperature, which is caused by the jump of compression ratio owing to the fluctuation of pressure of air storage tank, should also be taken into account.

According to the basic knowledge, power loss of multistage compression is lower than that of single-stage, and so does the outlet air temperature. It is suggested to optimize the whole system parameters including compressor capacity, stage number, mass flow rate, outlet air temperature, and charging time in an integrated manner, and evaluate the performance of air compression subsystem by considering indices, such as power loss and air temperature of outlet of compressor.

\subsection{Air storage subsystem}

The capacity of air storage subsystem determines the total capacity of the system, which is a key technology to implement the large-scale storage of high-pressure air. Large-scale CAES plants generally use underground salt cavern or manually excavated underground cave to store compressed air [31]. Regardless of transformation or completely manual excavation, cavern gas storage imposes harsh requirements on the geographical conditions. The originally planned Iowa CAES project is terminated due to its porous sandstone condition. Apparently, the choice of air storage space becomes a serious factor restricting the promotion of CAES [5, 32, 33].

In the design of small-scale CAES, pressure vessels, such as steel high-pressure tank and pipeline steels, are used to store high-pressure air, which can reduce its dependency on geographical conditions and realize a flexible layout $[34,35]$. Although, the high-pressure tank in market has reached the allowable pressure standard, its high construction cost makes it hard to be applied in the large-scale CAES system.

Fortunately, we can store air in pipeline steel, which is commonly used in natural gas transportation. Pipeline steel refers to the hot rolled coiled sheet or heavy plate for large diameter welded pipe used in oil and gas transportation [36]. Since the 1960s, with the continual upgrading, pipeline steel has been applied in long-distance pipeline transmission project worldwide, among which the laid X80 pipeline steel has reached more than $4300 \mathrm{~km}$, and related technology is very mature $[37,38]$. The pipeline steel technology has also been implemented to some major projects, such as the West-East Gas Delivery project in China. Compared with constructing high-pressure air storage tank, the pipeline steel method can significantly reduce the system cost, which is of great value for the commercial utilization of CAES technique in the site even without cavern. The operation pressure of the CSHC-100 NSF-CAES system is $8 \mathrm{MPa}$. This system has validated the engineering feasibility of pipeline steel based air storage technique.

Regarding the forms of air storage, the method to store high-pressure air directly is utilized in both traditional CAES and AA-CAES. However, this method is limited due to the low air storage capacity. The LAES system uses liquid air as the storage medium, greatly increasing the energy storage capacity and reducing the air storage space and storage cost. Therefore, LAES technique has the potential of massive promotion and application. Air storage subsystems of some typical CAES plants are illustrated in Table 2.

\subsection{Heat regeneration subsystem}

To improve power generation efficiency, SF-CAES adopts fossil fuel to heat air at the entrance of turbine. This kind of system has good technical maturity, and it is available to use the current mature technological products. The output energy of the system can be triple of using gas turbine alone if the process of recycling the waste thermal of tail air is utilized [4, 30, 39]. However, SF-CAES has strong fuel dependence and environmental pollution problems, which opposites the requirement of low-carbonemission.

Table 2 Air storage technique of typical CAES plants

\begin{tabular}{llll}
\hline Project & $\begin{array}{l}\text { Power } \\
(\mathrm{MW})\end{array}$ & Storage technique & Air state \\
\hline Huntorf & 321 & $310000 \mathrm{~m}^{3}$ salt cavern & High pressure air \\
McIntosh & 110 & $538000 \mathrm{~m}^{3}$ salt cavern & High pressure air \\
ADELE & 90 & Salt cavern & High pressure air \\
TICC-500 & 0.5 & Air storage tank & High pressure air \\
Sustain X & 1.5 & Pipe-type air storage & High pressure air \\
Highview & 0.35 & Air storage tank & Liquid air \\
LightSail & 0.5 & Steel tube & High pressure air \\
CSHC-100 & 0.1 & Pipeline steel & High pressure air \\
\hline
\end{tabular}




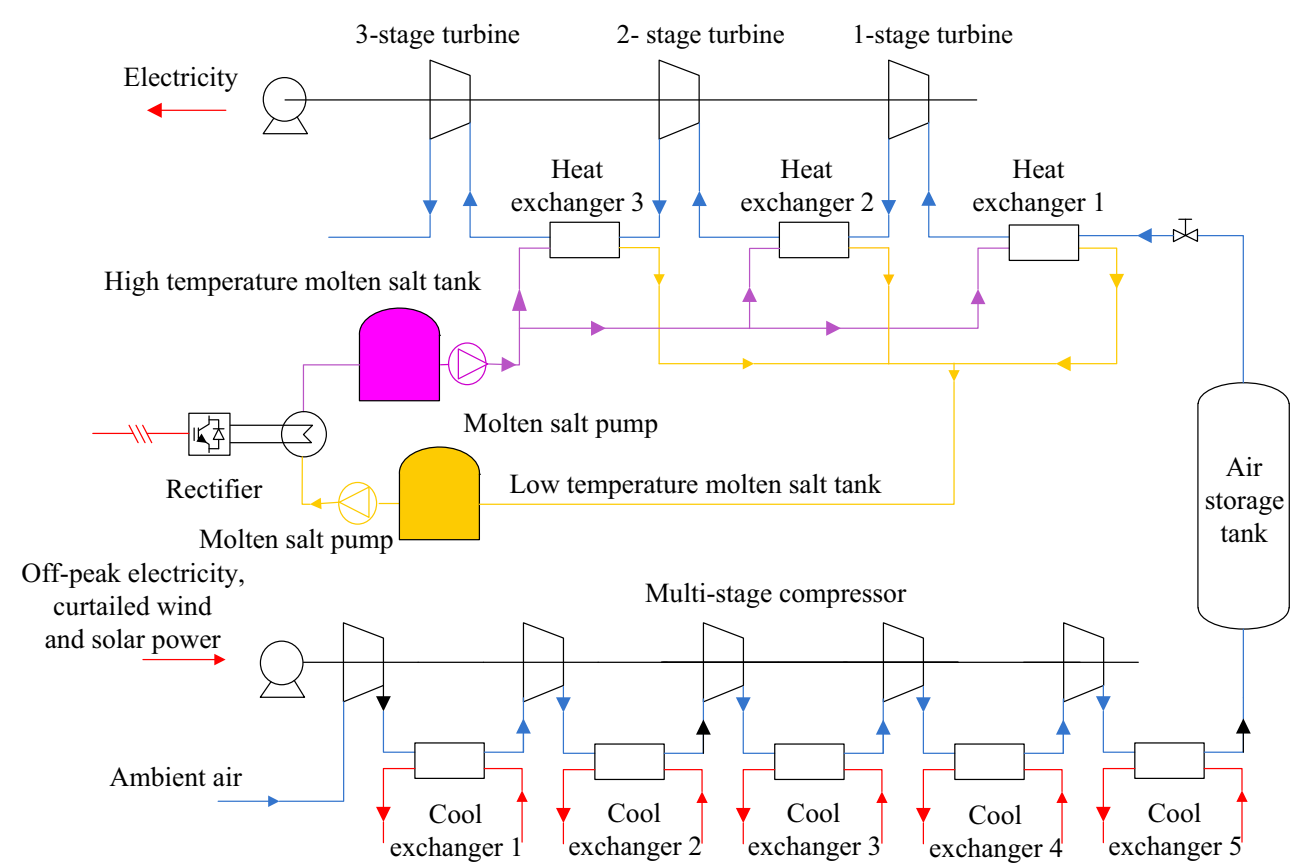

Fig. 4 Flow chart of molten salt based NSF-CAES system

Heat regeneration technology, one of the most important key technologies of NSF-CAES, is utilized to solve the above limitations of SF-CAES. Heat regeneration technology refers to the technique storing compression thermal and utilizing it to heat air entering each stage of turbine. This technology can be divided into single-stage and multistage heat regeneration with adiabatic compression based on heat regeneration approaches. Compared with the single-stage, the multi-stage scheme is more mature, of which each stage pressure ratio is more close to that of the current mature industrial compressor. Each stage compression is similar to that of the adiabatic compression, resulting in a more efficient compression performance.

Thermal energy source and TES carrier are two key factors of the heat regeneration subsystem. The heat regeneration technique has a major influence on the efficiency of the whole CAES system. The higher the temperature of the carrier of heat regeneration system is, the higher the system efficiency will be. Thus, it is important to select proper TES carrier. Most of the established CAES systems utilize the recycle water as the thermal storage carrier. Different interface forms of air and water, including spraying, foaming and bubbling, utilize in different systems [40]. For instance, Sustain X Company utilizes the foaming form, and LightSail Energy adopts the spraying form.

Although circulating water is cheap and easy to be obtained, the temperature range is small, and has a high dependence on the high-temperature compressor, so the performance is also limited. A feasible solution would be using molten salt or thermal oil as the thermal carrier in the heat regeneration subsystem. Bi-tank deployment scheme is also widely used in solar thermal generation system [41]. The wide implementation of molten salt and thermal oil owes to the superiority of heat transmission and thermal storage performance [42-45]. Thermal oil is usually used when the temperature is lower than $400{ }^{\circ} \mathrm{C}$, and is deployed with special devices including pressure valve, whereas molten salt has a higher operation temperature range, and is easy to be implemented. A typical molten salt based 5-stage compression and 3-stage expansion NSFCAES system is illustrated in Fig. 4. The CSHC-100 uses the thermal oil as thermal energy carrier.

\subsection{Turbine generation subsystem}

Turbine generation subsystem is the key component of realizing thermoelectric conversion during energy discharging process, and the efficiency of this subsystem determines the efficiency of the whole CAES system. Considering that the structure of turbine similar to that of gas generator, most of the current CAES systems utilize the architecture of multi-stage expansion and reheat in the middle. Micro CAES can use turbine components of the micro gas turbine, reciprocating expansion engine, or screw type air engine. However, the technology involved is not yet mature, and still in the improvement periods $[46,47]$. 
The small-scale CAES has a relatively lower power output, leading to a lower air flow. Therefore, the impeller is relatively smaller and the rotation rate of the turbine is higher. Meanwhile, the expansion of turbine will be greater due to the demand of increasing the energy storage capacity per gas storage volume unit, causing the decline in the specific enthalpy-drop ratio of the air. In this case, the linear velocity of wheel circumference of the impeller rises, indicating the increase in the rotation rate of the turbine. Based on the two factors mentioned above, the rotation rate of the turbine in kilowatt CAES can reach above the level of tens of thousands. Therefore, the turbine generation subsystem of traditional turbine expander needs to be equipped with speed reducers $[48,49]$. For instance, TICC-500 utilizes a speed reducer with a 20:1 ratio [12]. High-speed gear reducer needs to be designed due to the huge difference in rotation rate between turbines and conventional synchronous generators. However, it has limitations in stability, service life and loss in the process of high-speed rotation, vibration and noise. Besides, it also needs to be configured with complex oil supply system [50-52].

With the development of high-speed motor technology, the utilization of generator with high-speed direct drive turbo expander is becoming a new development direction. Through the adaptation of high-speed generator solution, the overall system has advantages in volume, weight, construction costs, and operating efficiency. It is more suited to satisfy the need of variable parameter operation of turbine expansion process [51-53]. However, development of the design of high-speed motor, rotor dynamics analysis, gas bearings technology, and corresponding power electronic devices are still in the initial stage. An in-depth study is needed.

The CSHC-100 NSF-CAES utilizes the high-speed motor. The system adopts interior permanent magnet rotor structure to effectively handle issues of the magnetic bridge to bear the centrifugal force and reduce reluctance torque. Besides, for the rapid dissemination of heat generated by eddy currents, the rotor can be coated on the surface layer of heat-emitting material and the heat released by emitting infrared, the heat of the rotor is transmitted to the stator housing vacuum environment.

Currently, the research of power electronic converter for adjusting the output voltage of the high-speed motor and corresponding control technology is more mature, so the high-speed motor-based small-scale CAES technology is very attractive.

\section{CAES application prospects}

There are many challenges in smart grid, such as the increasing gap of peak and valley load, the increasing uncertainty of sources and loads and the multiple energy carriers' conversion needs in the energy internet. In these application scenarios, NSF-CAES has many advantages, such as the capabilities of spatial and temporal power shifting, cold-heat-power tri-generation, zero carbon emission, high efficiency, off-the-shelf technologies, convenient equipment manufacturing, short construction period, and low investment risk. Therefore, NSF-CAES is undoubtedly destined to create great value in the construction of smart grid and energy internet.

\subsection{Improve flexibility of smart grid}

\section{1) Power load shifting}

The new generation of centralized NSF-CAES plant has a capacity more than $50 \mathrm{MW}$, and is capable of supplying power up to several hours, which is higher than the conventional battery energy storage with 1 to 2 orders of magnitude. Constructing large-scale centralized NSFCAES power plant in the area with mined salt caverns can promote the economic operation of power systems by consuming surplus off-peak electricity and supplying power for the on-peak hours to shift power load [54].

Small-scale decentralized NSF-CAES has a capacity up to $1-50 \mathrm{MW}$, which has high flexibility. It can be used for the load shifting of distribution network and micro-grid. Off-peak power can be used for charging and supplying onpeak load to achieve economic operation of the micro-grid and power distribution network.

\section{2) Renewable energy consumption}

Needless to say, how to consume renewable energy under the condition of the safe and reliable operation of power system is a serious problem. In recent years, severe wind and solar curtailment phenomenon in Northeast and Northwest China indicates an emergent need for energy storage devices [55]. In this regard, CAES will provide new opportunities for the effective consumption of renewable energy including wind and solar [56].

The centralized NSF-CAES system can be deployed with a large, centralized wind or photovoltaic power base, to construct coupled wind-storage or wind-solarstorage system. The NSF-CAES can stabilize the volatility of a centralized renewable energy base to make it friendly connected to the grid. The NSF-CAES system can also reduce the renewable curtailment by enabling large-scale effective consumption of wind and solar power [57].

The new generation of distributed NSF-CAES system uses steel material based tank like pipeline steel as air storage reservoir. The construction of this kind of system has a very low requirement for the external environment and resource, and it can utilize rich air as the main 
medium. The distributed NSF-CAES can be employed with a megawatt capacity, combined with photovoltaic power plants as CSHC-100, wind farms, hydropower and other supporting construction, to build wind power and CAES hybrid system [58]. Implemented with wind, solar, hydro, and storage cooperative self-autonomy control [59], NSF-CAES can greatly decrease the current wind, solar and water power curtailment phenomenon [60]. In particular, the distributed NSF-CAES system can be implemented to independent power system in the desert, mountains, islands, and other remote locations or harsh environments. By integrating NSF-CAES with wind power, solar power, tidal power, and other clean energy sources, it is expected to build a low-carbon combined cooling-heating-power supply independent power systems, and stabilize the fluctuation of photovoltaic, wind power and tidal power, and provide electricity stably and reliably [61].

\subsection{Construction of energy internet}

Based on the energy internet or the integrated energy system, the utilization of wind, solar, water, natural gas, and other clean energy sources in an integrated manner is a consensus to address the global energy crisis and environmental pollution problems. Energy internet hub acts as the interface among the power network, the heating network and the gas pipeline network, and can be implemented to the integrated optimization and control among multi-energy networks $[62,63]$. Energy hub is required to capability of energy conversion, storage and transmission $[62,63]$. Besides, a clean energy hub is expected to produce no-carbon emission during service.

NSF-CAES uses compressed thermal energy recycling system to replace the fuel supplementary fired subsystem in SF-CAES. The decoupling storage of molecular potential energy of high-pressure air and thermal energy and the capability of joint supplying of combined cooling-heating-power make it a very attractive clean energy hub [64]. Concentrated NSF-CAES can be used to the construction of energy internet, and realize the integrated utilization of various energy sources. On the other hand, distributed small scale NSF-CAES can gather electricity, gas, heat, and other resources together to construct a micro energy internet in urban and rural communities, industrial park, factories, schools, and other places. Through conversion among multi-carriers, integrated dispatch of all energy and coordinated planning are realized to reduce the system operation cost. The architecture of NSF-CAES based clean energy hub is illustrated in Fig. 5. The hub mainly includes energy conversion,

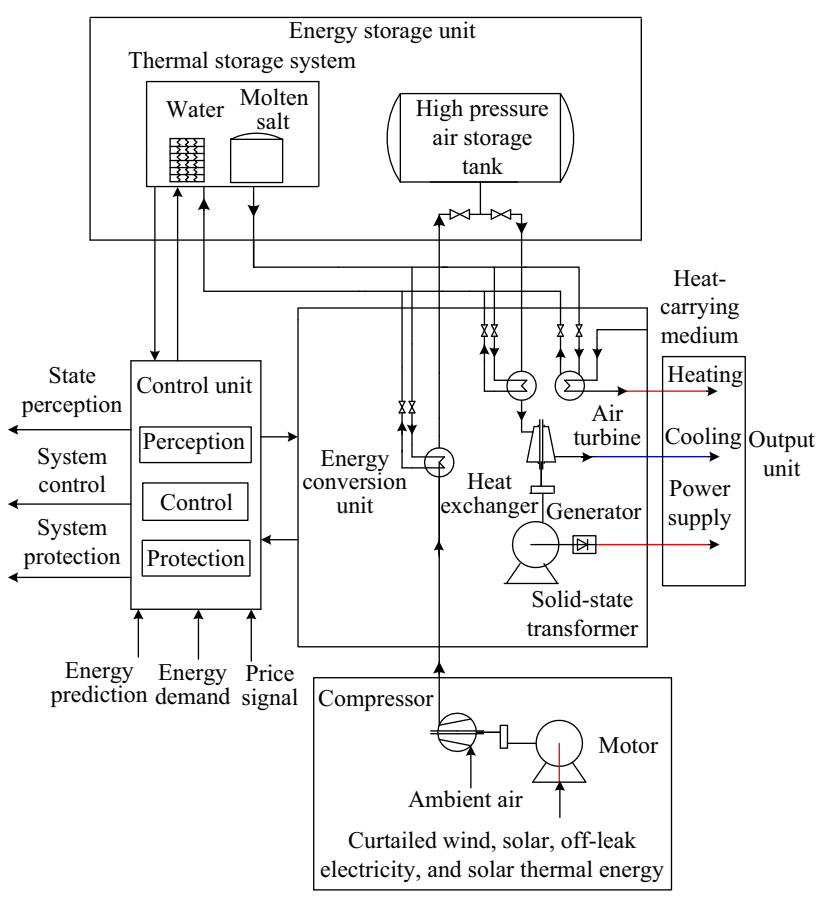

Fig. 5 Architecture of NSF-CAES based clean energy hub

energy output and control unit. More details of each unit can be found in [64].

\section{Factors restricting development of CAES and possible resolutions}

At present, China is the world largest wind and solar power producer. However, the progress of storage construction has fallen behind. Energy storage technology development level has become an important indicator to measure the development level of a nation's renewable energy industry. As a large-scale energy storage technology, CAES has the advantages of large storage capacity, long operation life, non-pollution and so on, and it has a wide application prospects. But the energy storage efficiency, system cost and other factors put a brake on the further development of CAES. Developing CAES system with high efficiency and low cost is an urgent problem to be solved. Meanwhile, it is important to establish and perfect the corresponding energy storage market mechanism for the healthy development of the energy storage technology.

\subsection{Energy storage efficiency}

NSF-CAES system is generally composed of air compression subsystem, air storage subsystem, heat regeneration subsystem, and turbine generation subsystem. Energy storage efficiency of the system is closely related to each 
subsystem. So the energy efficiency of the system can be enhanced by improving the performance of any subsystem.

1) As the core facility in the compression process, the compressor determines the efficiency of the energy storage process. According to the needs of future CAES system, compression technology of large air flow, high efficiency and high exhaust temperature will be developed. By increasing the exhaust temperature of the compressor, the heat storage temperature of the system can be improved, so can the energy storage efficiency of the system.

2) The conventional CAES can use the air as energy storage medium, and require the air storage system with a large capacity and persistent high-pressure. As a large-scale air storage technology, underground salt cavern meets the requirements of CAES. Due to a large amount of air stored in salt cavern, the pressure fluctuation of the gas storage chamber is small, which significantly improves the energy efficiency of the system.

For the CAES power generation station without having the salt cavern gas storage condition, the natural gas pipeline transportation technology can be employed. This technology can save a more than $50 \%$ cost compared with the conventional high-pressure gas storage container, which can greatly expand the application prospects of CAES.

3) The parameters of the heat regeneration subsystem have great influence on the efficiency of NSF-CAES system. The higher the temperature is, the higher the efficiency of ESS will be. The overall energy efficiency of the system can be improved by increasing the storage temperature and efficiency of the heat regeneration subsystem.

In the area with abundant solar resources, the solar energy can be utilized as the heat source of the heat regeneration subsystem. The main idea is based on trough collector and high-temperature heat storage technology, using solar energy to increase the turbine inlet air temperature, so as to enhance the system operation ability and power efficiency of energy storage.

4) Turbine generation system is the core component for thermoelectric conversion in energy discharging process. Its efficiency directly determines the overall efficiency of ESS. Currently, there is no specialized large-scale air turbine. Therefore, according to the thermal characteristic of air, the development of new and efficient air turbine is the key to improving the efficiency of the expansion power generation system.

5) Considering the system cycle, improving the thermal process and exploring new generation system are important ways to enhance the storage efficiency of the system. Especially for LAES, it is the key to ensuring the energy storage efficiency of the system, which is a more efficient liquefaction and cold storage process.

\subsection{Construction cost}

The key to save the cost of CAES is to reduce the cost of each subsystem, especially the TES system and gas storage system.

1) To reduce the construction cost of the system, heat storage and cold storage will become important aspects of the research. Low cost heat storage/cold storage working fluid is desired. For the high-temperature TES system, the light-heat storage technology should be referenced. The mature storage technology is used to reduce the cost of the system.

2) The low cost storage technology should be developed. Using the underground salt caverns and the special geological conditions of the cave, mine is helpful in reducing the construction cost. As a mature technology of large capacity storage, salt cavern gas storage has been successfully exploited in natural gas storage. The storage pressure and scale meet the CAES usage, and its cost is much lower than the artificial pressure container.

3) For small-scale CAES, advanced electric and electronic technology are used to fulfill the direct connection of high-speed turbine to generator, remove the mechanical reducer, simplify the structure of the system, and improve the system reliability. By using the advanced power electronics technology, it also helps to reduce the construction cost of CAES.

\subsection{Market mechanism}

The sustainable development of energy storage technique is inseparable from the effective market mechanism. The development and application of CAES system in smart grid and energy internet need more supports of appropriate incentives, policies, regulations, and market trading mechanism.

Proper energy storage pricing mechanisms and policies are important factors to support the sustainable development of the energy storage industry. It is also an important measure to guide the ESS to participate in power grid ancillary services. Energy pricing should take full account of the ESS and the potential benefits. In short, energy storage benefits should not be limited in the output power of ESS, the benefits of the need scenery 
electricity storage to increase the hours and reduced carbon emissions, and other potential benefits are also should be considered, so as to improve the energy pricing mechanism and guide the storage industry sustained and stable development.

With the improvement of regulation policies and market incentives, a new generation of CAES system, which is advantageous in its large capacity, high efficiency and cleanness, will play an increasingly important role in future energy systems.

\section{Conclusion}

As a promising energy storage technique, CAES has great potential for development. Facing the future energy field, this paper focuses on the basic principles, technical characteristics of key subsystems, as well as the application of CAES technology. Meanwhile, possible resolutions for the application prospects and the development direction of CAES technology are suggested. To promote energy internet construction, energy conservation and emission reduction, we can use major science and technology projects as the carrier, seeking for collaboration among the government agency, industry and academia, and research institutes, start from different thinking of large-scale and small-scale CAES, thus greatly leveraging the crucial development of CAES industry.

Acknowledgments This work was supported by National Natural Science Foundation of China (No. 51321005).

Open Access This article is distributed under the terms of the Creative Commons Attribution 4.0 International License (http:// creativecommons.org/licenses/by/4.0/), which permits unrestricted use, distribution, and reproduction in any medium, provided you give appropriate credit to the original author(s) and the source, provide a link to the Creative Commons license, and indicate if changes were made.

\section{References}

[1] Tan ZF, Ngan HW, Wu Y et al (2013) Potential and policy issues for sustainable development of wind power in China. J Mod Power Syst Clean Energy 1(3):204-215. doi:10.1007/ s40565-013-0037-8

[2] Xie L, Carvalho PM, Ferreira LAFM et al (2011) Wind integration in power systems: operational challenges and possible solutions. Proc IEEE 99(1):214-232

[3] Beaudin M, Zareipour H, Schellenberglabe A et al (2010) Energy storage for mitigating the variability of renewable electricity sources: an updated review. Energy Sustain Dev 14(4):302-314

[4] Chen HS, Cong TN, Yang W et al (2009) Progress in electrical energy storage system: a critical review. Prog Nat Sci 19(3):291-312
[5] Inage S (2009) Prospects for large-scale energy storage in decarbonised power grids. International Energy Agency (IEA), Paris

[6] Wang CS, Wu Z, Yang XS et al (2014) Modeling and verification of hybrid energy storage system based on micro compressed air energy storage. Autom Electr Power Syst 38(23):22-26. doi:10.7500/AEPS20131104010

[7] Lemofouet S, Rufer A (2006) Hybrid energy storage systems based on compressed air and supercapacitors with maximum efficiency point tracking. IEEE Trans Ind Electron 53(4):1105-1115

[8] Wang C, Chen LJ, Liu F et al (2014) Thermal-wind-storage joint operation of power system considering pumped storage and distributed compressed air energy storage. In: Proceedings of the 2004 power systems computation conference (PSCC'14), Wroclaw, Poland, 8-22 August 2014, 7 pp

[9] Díaz-González F, Sumper A, Gomis-Bellmunt O et al (2012) A review of energy storage technologies for wind power applications. Renew Sustain Energy Rev 16(4):2154-2171

[10] Chai L (2014) Research progress of cold storage and thermal storage device for supercritical air energy storage system. Institute of Engineering Thermophysics, Chinese Academy of Sciences, Beijing. http://www.etp.ac.cn/xwdt/kydt/201403/ t20140307_4047538.html. Accessed 6 Sep 2016

[11] Hu S (2016) 863 "supercritical compressed air energy storage system research, development and demonstration" subject through technical acceptance. ESCN, Beijing. http://www.escn. com.cn/news/show-324452.html. Accessed 6 Sep 2016

[12] Mei SW, Wang JJ, Tian F et al (2015) Design and engineering implementation of non-supplementary fired compressed air energy storage system: TICC-500. Sci China E 58(4):600-611

[13] Luo X, Wang JH, Dooner M et al (2014) Overview of current development in compressed air energy storage technology. Energy Procedia 62:603-611

[14] Chen HS, Liu JC, Guo H et al (2013) Technical principle of compressed air energy storage system. Energy Storage Sci Technol 2(2):146-151

[15] Crotogino F, Mohmeyer KU, Scharf R (2001) Huntorf CAES: more than 20 years of successful operation. In: Proceedings of the Solution Mining Research Institute (SMRI) spring meeting, Orlando, FL, USA, 15-18 April 2001, 8 pp

[16] Succar S, Williams RH (2008) Compressed air energy storage: theory, resources, and applications for wind power. Princeton Environmental Institute, Princeton

[17] Huntorf CAES Powerplant. http://enipedia.tudelft.nl/wiki/ Huntorf_Caes_Powerplant. Accessed 12 June 2016

[18] Schulte RH, Critelli N Jr, Holst K et al (2012) Lessons from Iowa: development of a 270 megawatt compressed air energy storage project in midwest independent system operator: a study for the DOE energy storage systems program. Sandia National Laboratories, Albuquerque

[19] John F (2013) FirstEnergy postpones project to generate electricity with compressed air. http://www.cleveland.com/business/ index.ssf/2013/07/firstenergy_postpones_project.html. Accessed 12 June 2016

[20] Budt M, Wolf D, Span R et al (2016) A review on compressed air energy storage: basic principles, past milestones and recent developments. Appl Energy 170:250-268

[21] ADELE Isothermal CAES. http://enipedia.tudelft.nl/wiki/ Adele_Isothermal_CAES. Accessed 6 Sep 2016

[22] Highview Power Storage. http://www.highview-power.com/ tour/. Accessed 12 June 2016

[23] Lightsail Energy. http://www.lightsail.com. Accessed 12 June 2016

[24] SustainX. http://www.sustainx.com. Accessed 12 June 2016 
[25] Supercritical compressed air energy storage system development. Chinese Academy of Sciences, Beijing. http://www.cas. cn/ky/kyjz/201307/t20130724_3904638.shtml. Accessed 6 Sep 2016

[26] Wang SX, Zhang XL, Yang LW et al (2016) Experimental study of compressed air energy storage system with thermal energy storage. Energy 103:182-191

[27] Liu B, Chen LJ, Mei SW et al (2014) The impact of key parameters on the cycle efficiency of multi-stage RCAES system. J Mod Power Syst Clean Energy 2(4):422-430. doi:10. 1007/s40565-014-0090-y

[28] Greenblatt JB, Succar S, Denkenberger DC et al (2007) Baseload wind energy: modeling the competition between gas turbines and compressed air energy storage for supplemental generation. Energy Policy 35(3):1474-1492

[29] Kurz R, Brun K, Meher-Homji C et al (2012) Gas turbine performance and maintenance. In: Proceedings of the 41 turbomachinery symposium, Houston, TX, USA, 24-27 September 2012, $32 \mathrm{pp}$

[30] Heavy duty gas turbine products (2009). GE Energy, Atlanta

[31] Zhang XJ, Chen HS, Liu JC et al (2012) Research progress in compressed air energy storage system: a review. Energy Storage Sci Technol 1(1):26-40

[32] Barnes FS, Levine JG (2001) Large energy storage systems handbook. CRC Press, Boca Raton

[33] Oberhofer A, Meisen P (2012) Energy storage technologies and their role in renewable integration. Global Energy Network Institute (GENI), San Diego

[34] Penberton D, Jewitt J, Pletka R (2008) Mini-compressed air energy storage for transmission congestion relief and wind shaping applications. New York State Energy Research and Development Authority, Albany

[35] Bi JT, Jiang T, Chen WL et al (2013) Research on storage capacity of compressed air pumped hydro energy storage equipment. Energy Power Eng 5(4):26-30

[36] Yin YQ, Lei XW, Wu KM (2012) Current status and prospectus of ultra-high strength pipeline steel development. China Met 22(9):5-9

[37] Maes MA, Dann M, Salama MM (2008) Influence of grade on the reliability of corroding pipelines. Reliab Eng Syst Saf 93(3):447-455

[38] Zhang CG, Zheng L, Xie SQ et al (2014) Recent developments of large diameter X80 UOE line pipes. Baosteel Tech Res $8(2): 46-54$

[39] Eckroad S, Gyuk I (2003) EPRI-DOE handbook of energy storage for transmission and distribution applications. Electric Power Research Institute, Inc., Palo Alto

[40] McBride T, Bell A, Kepshire D (2013) ICAES innovation: foam-based heat exchange. SustainX, Seabrook

[41] Wu YT, Zhang LN, Ma CF (2007) High temperature thermal energy storage technology of solar thermal generation. Sol Energy 3:23-25

[42] Agyenim F, Hewitt N, Eames P et al (2010) A review of materials, heat transfer and phase change problem formulation for latent heat thermal energy storage systems (LHTESS). Renew Sustain Energy Rev 14(2):615-628

[43] Ren T, Liu S, Yan GC et al (2016) Temperature prediction of the molten salt collector tube using BP neural network. IET J Mag 10(2):212-220

[44] Li SD, Yan GF, Fu XD et al (2013) The state-of-art of thermal oil applied in mid-and high-temperature solar heat utilization system. Mater Rev 27(9):10-13

[45] Kalogirou SA (2004) Solar thermal collectors and applications. Prog Energy Combust Sci 30(3):231-295

[46] Review of electrical energy storage technologies and systems and of their potential for the UK (2004). EA Technology, Chester
[47] Zuo CJ, Qian YJ, An D et al (2007) Experimental study on airpowered engine. Chin J Mech Eng 43(4):93-97

[48] Kaplan U (2007) Organic rankine cycle configurations. In: Proceedings of the 2007 European geothermal congress, Unterhaching, Germany, 30 May-1 June 2007

[49] Marcuccilli F, Zouaghi S (2007) Radial inflow turbines for Kalina and organic rankine cycles. In: Proceedings of the 2007 European geothermal congress, Unterhaching, Germany, 30 May-1 June 2007

[50] Wang FX (2006) Study on design feature and related technology of high speed electrical machines. J Shengyang Univ Technol 28(3):258-264

[51] Sun LJ, Ren XK, Gao YJ et al (2015) Study on feature and related technology of high speed turbine generator. Cryog Supercond 43(8):23-26

[52] Wu Y, Zhang HT, Huang H et al (2014) Comparison and analysis of engineering application of two types of liquid turbine expander. Cryog Technol 5:23-25

[53] Bianchi N, Bolognani S, Luise F (2004) Potentials and limits of high-speed PM motors. IEEE Trans Ind Appl 40(6):1570-1578

[54] Salgi G, Lund H (2008) System behaviour of compressed-air energy-storage in Denmark with a high penetration of renewable energy sources. Appl Energy 85(4):182-189

[55] Li JF, Cai FB, Qiao LM et al (2014) 2014 China wind power review and outlook. Chinese Renewable Energy Industries Association (CREIA), Beijing, China; Chinese Wind Energy Association (CWEA), Beijing, China; Global Wind Energy Council (GWEC), Brussels

[56] Lund H, Salgi G (2009) The role of compressed air energy storage (CAES) in future sustainable energy systems. Energy Convers Manag 50(5):1172-1179

[57] Arabkoohsar A, Machado L, Koury RNN (2016) Operation analysis of a photovoltaic plant integrated with a compressed air energy storage system and a city gate station. Energy 98:78-91

[58] Saadat M, Shirazi FA, Li PY (2014) Revenue maximization of electricity generation for a wind turbine integrated with a compressed air energy storage system. In: Proceedings of the 2014 American control conference (ACC'14), Portland, OR, USA, 4-6 June 2014, pp 1560-1565

[59] Lu Q, Sheng CY, Chen Y (2011) Coordinated autonomous control strategy for power systems with large-scale wind power plants. Control Theory Appl 28(10):1491-1495

[60] Wan C, Zhao J, Song YH et al (2015) Photovoltaic and solar power forecasting for smart grid energy management. CSEE J Power Energy Syst 1(4):38-46

[61] Cai GL, Chen RY, Chen JB (2012) Research on simulations and model of the isolated wind-solar-diesel-battery hybrid microgrid. In: China international conference on electricity distribution (CICED), pp 1-4

[62] Shabanpour-Haghighi A, Seifi AR (2015) Energy flow optimization in multicarrier systems. IEEE Trans Ind Inform 11(5):1067-1077

[63] Geidl M, Andersson G (2007) Optimal power flow of multiple energy carriers. IEEE Trans Power Syst 22(1):145-155

[64] Xue XD, Mei SW, Lin QY et al (2016) Energy internet oriented non-supplementary fired compressed air energy storage and prospective of application. Power Syst Technol 40(1):164-171

Laijun CHEN received the B.Eng. and Ph.D. degrees, both in electrical engineering, from Tsinghua University, Beijing, China in 2006 and 2011, respectively. He is currently an associate professor in the Department of Electrical Engineering, Tsinghua University. He is also the vice director of New Energy (Photovoltaic) Industry Research Center, Qinghai University. His research mainly concerns distribution generation, microgrids and energy storage. 
Tianwen ZHENG received the Ph.D. degree in electrical engineering, from Tsinghua University, Beijing, China in 2016. Now he is engaged in postdoctoral research in Tsinghua University. His research interests include control and optimization of distributed energy system.

Shengwei MEI received the B.S. degree in mathematics from Xinjiang University, Urumqi, China, received the M.S. degree in operations research from Tsinghua University, Beijing, China, and received the $\mathrm{Ph} . \mathrm{D}$. degree in automatic control from the Chinese Academy of Sciences, Beijing, China in 1984, 1989, and 1996, respectively. He is currently a professor of Department of Electrical Engineering at Tsinghua University. His research interests include power system analysis and control, compressed air energy storage system, engineering game theory, and power grid complexity.

Xiaodai XUE received the Ph.D. degree in engineering from Chinese Academy of Sciences in 2012. Now he is engaged in postdoctoral research in Tsinghua University. His research interests include compressed-air energy storage, thermodynamic system design and solar thermal system.
Binhui LIU received the B.S. degree in electrical engineering from Tsinghua University in 2016. She is currently pursuing the Master's degree in electrical engineering in Tsinghua University, Beijing, China. Her current research interest includes compressed air energy storage system.

Qiang $\mathbf{L U}$ received the B.S. degree in electrical engineering from the Department of Electrical Engineering at Tsinghua University, Beijing, China, in 1959. He is currently a professor of Tsinghua University. He is an academician of the Chinese Academy of Sciences. His research interests include power system analysis and control, smart grid, and compressed air energy storage system. 